\title{
The poor outcome of conduct disorders: a need for innovative, more effective therapeutic interventions
}

\author{
Alessandro Zuddas
}

Published online: 26 June 2014

(C) Springer-Verlag Berlin Heidelberg 2014

Conduct disorder (CD) is defined as a repetitive and persistent pattern of behaviour, which violates the rights of others and major age-appropriate societal rules. According to DSM 5, a diagnosis of CD requires the presence of 3 of 15 criteria that should have been present in the last 12 months, one of which must have been present in the past 6 months. These 15 behavioural criteria are categorized into 4 behavioural dimensions: aggression to people and animals, destruction of property, deceitfulness or theft, and serious violation of rules such as running away form home; in order to formulate the diagnosis of $\mathrm{CD}$, behavioural symptoms must cause clinically significant impairment in social, academic or occupational functioning [1]. It has been suggested that the diagnosis should be valid only when symptoms are due to an "internal dysfunction" of the individual, rather than to only a transient reaction to a negative environment [2]. Multiple causal factors (e.g. genetic, neurocognitive, temperamental, peer, family) underlie the behavioural manifestations of CD: geneenvironment interactions appear to play a crucial role in the development of the disorder [3, see also 4 for a review].

In the last decades, specific pathways associated with the emergence and continuity of $\mathrm{CD}$ over time have been extensively investigated. A seminal article published by Moffit in 1993 [5] indicated that two groups of patients can be differentiated by the courses, correlates and causes of their antisocial behaviour: (1) a life-course persistent

\footnotetext{
A. Zuddas $(\square)$

Department of Biomedical Sciences, University of Cagliari,

Cagliari, Italy

e-mail: azuddas@unica.it

A. Zuddas

Child \& Adolescent Neuropsychiatry, "Microcitemico"

Paediatric Hospital, Via E. Jenner, 09121 Cagliari, Italy
}

(LCP) group with stable high levels of aggression and antisocial behaviour starting in childhood and continuing into adulthood, and (2) an adolescence-limited (AL) group of patients, whose antisocial behaviours are primarily nonaggressive, and who adopt pro-social roles and engage in more mature decision-making during the transition into adulthood. In Moffitt's taxonomy, early CD was related to neuropsychological deficits (i.e. deficit in inhibitory control, poor verbal abilities), leading to difficulties managing peer conflicts, regulating emotions and controlling impulses: no neuropsychological impairments were reported in the AL group. LCP was, therefore, considered the neurodevelopment disorder deriving from the interaction between individual vulnerability and environmental adversities, whereas the $\mathrm{AL}$ was considered more a social mimicry of deviant peers than a true neurobiological disorder.

More recent studies, several by the Moffitt's group, challenged this two-category taxonomy: a childhood-limited trajectory has been characterized as a frequent form of disruptive behaviour $[6,7]$ and an adolescent persistent pathway has been observed in girls (who rarely show a childhood-onset presentation) as well as in boys. Moreover, a significant impairment in neuropsychological functioning (i.e. facial emotion recognition measured by fMRI) was observed in adolescent patients with both childhood and adolescent onset $[8,9]$.

Over the past few years, the limited pro-social emotions expressed by a significant percentage of adolescents with $\mathrm{CD}$ have been extensively investigated: the neurobiology of the callous-unemotional traits (i.e. lack of concern for others' feelings, deficient guilt and remorse, and shallow affect and empathy) and their interference with socialization and with the available therapeutic intervention has been established [10, see also 11 for a review]. 
Interestingly, in adolescent girls both affective and cognitive empathy appears impaired, whereas in males callous-unemotional traits may be observed mainly in the childhood-onset subtype, involving affective more than cognitive empathy [12] with corresponding significant differences in neuroimaging [9].

A recent review by Fairchild [13] summarizes many of these recent findings, suggesting that differences between LCP and adolescent-onset forms of antisocial behaviour appear to be quantitative rather than qualitative and that adolescent-onset antisocial behaviour may also be a neurodevelopmental disorder, often leading to significant impairment in adult life.

Following these lines, the study of Kretschemer et al. [14 in this issue] based on a large general population sample (7,218 individuals from the Avon longitudinal study), rather than a high-risk sample as in several other outcome studies, tested whether childhood conduct problem trajectories are predictive of a wide range of other health and behaviour problems in early adulthood. Using a sophisticated statistical approach, including latent class growth analysis and a set of logistic regression models, the authors identified four developmental trajectories [the four classes were defined as "low", meaning low level of behavioural symptoms, "childhood limited" (CL), "early onset persistent" (EOP) and "adolescent onset" (AO)]. The presence of conduct problems (the last three classes) was associated with the majority of the investigated poor outcomes. Individuals who displayed EOP were at greater risk for a large series of adulthood problems ranging form alcohol, tobacco, cannabis and other drug use to criminal offenses, self-harm, gambling, risky sexual behaviour to anxiety and depression. The EOP class was three times more likely than the "low" class to consume cannabis, and twice as likely to engage in smoking and risky sexual behaviour (results also observed for the AO class). Also, subjects of the CL trajectory showed a higher risk for smoking, but this effect disappeared when all the confounders (family and child characteristics, birth information and child experience) were taken into account.

The results of the study support the previous findings of an LCP group of individuals with stable, significantly impairing, problem behaviour involving a large range of contexts at different life stages, but they also indicate that the so-called adolescent-limited subtype is not just a temporary problem. As stated by the authors, and reported by others, adolescent-onset problem behaviour may be associated with delinquent peer involvement which in turn may lead to substance use, risky sexual behaviours, getting these youngsters trapped in maladjustment and persistent future problems. Unfortunately, only non-specific information is provided on a possible gender effect and on the presence and possible moderating effect of callous- unemotional traits: nevertheless, the results of the study strongly suggest that an accurate assessment and a personalized intervention are needed for all adolescents with serious conduct symptoms.

The recent outstanding academic research on conduct disorders has been rarely translated into clinical practice: in several European countries children and adolescents with $\mathrm{CD}$ do not receive specific help for their disorder. The recently published NICE guideline [15] indicates that the clinical assessments of children with severe and complex behavioural problems should be conducted by well-trained specialists and be comprehensive enough to identify coexisting mental health problems and complicating factors, and that multimodal treatment interventions may be effective for reducing antisocial and offending behaviour. Nevertheless, in many community developmental mental health services it is still a common perception that the younger children may outgrow their problems and older adolescents may best managed by non-health services within the community: the maladaptive behaviour is often considered a transient consequence of "poor parenting" rather than a genuine neurobiological developmental disorder [16]. It should also be pointed out that very little good-quality evidence supports the efficacy, effectiveness and safety of medication treatments for $\mathrm{CD}$; guidelines usually do not recommend pharmacological interventions for the disorder, apart from short-term risperidone in children and adolescents with subaverage IQ [17], leading to the consideration that at least part of this efficacy may be related to just sedation and decreasing reactivity and anxiety.

Taking the cost of specific neuropsychological interventions into consideration, the need for highly personalized and specific programs of care [18] contrasts with the poor evidence of efficacy of non-specific interventions, which are, however, provided by many community developmental mental health services. This discrepancy renders it possible that, at least in some countries diversion of funding and resources for CD to other mental health disorders may have occurred. Resources may have also been shifted to different non-health services, which are often unable to improve outcome and adjustment of these youngsters. The financial crisis involving the health system in many European countries is potentially leading to a further deterioration of this scenario.

Fortunately, the EU has recently granted funding to a series of large research projects on CD. The "Paediatric European Risperidone Studies" (PERS) study will investigate the efficacy and safety of risperidone in normal IQ children and young people with $C D$, who have an IQ in the normal range [19]. The "Aggression subtyping for improved insight and treatment innovation in psychiatric disorders" (AGGRESSOTYPE), the "Neurobiology and 
Treatment of Adolescent Female Conduct Disorder: The Central Role of Emotion Processing" (FEM-NAT CD) and the "Multidisciplinary Approaches to Translational Research In Conduct Syndromes" (MATRICS) programmes will each include nested projects, finalized to identify neural, genetic and molecular factors involved in the pathogenesis of aggression/antisocial behaviour in preclinical (animal) models and clinical samples, in order to develop and pilot- test preventive interventions in very young children at very high risk (i.e. those with callousunemotional traits), and to perform proof-of-concept clinical studies in order to identify innovative pharmacological interventions.

In the meantime, the dissemination of all the available information on the developmental, neurobiological nature of conduct disorders and the intense implementation of the guideline recommendations of an early recognition, accurate assessment, early evidence-based neuropsychological and multimodal interventions should be achieved by bidirectional communication and interaction between practitioners and researchers, with the crucial involvement of the local and national policy makers.

\section{References}

1. American Psychiatric Association (2013) Diagnostic and statistical manual of mental disorders, 5th edn. American Psychiatric Association, Washington, DC

2. Wakefield JC, Pottick KJ, Kirk SA (2002) Should the DSM-IV diagnostic criteria for conduct disorder consider social context? Am J Psychiatry 159:380-386

3. Caspi A, McClay J, Moffitt TE, Mill J, Martin J, Craig IW et al (2002) Role of genotype in the cycle of violence in maltreated children. Science 297:851-854

4. Buitelaar IK, Smeets CK, Herpers P, Scheepers F, Glennon J, Rommelse NJ (2013) Conduct disorders. Eur Child Adolesc Psychiatry 22(Suppl 1):S49-S54

5. Moffitt TE (1993) Adolescence-limited and life-course-persistent antisocial behavior: a developmental taxonomy. Psychol Rev 100:674-701

6. Odgers CL, Caspi A, Broadbent JM, Dickson N, Hancox RJ, Harrington $\mathrm{H}$ et al (2007) Prediction of differential adult health burden by conduct problem subtypes in males. Arch Gen Psychiatry $64: 476-484$

7. Odgers CL, Moffitt TE, Broadbent JM, Dickson N, Hancox RJ, Harrington $\mathrm{H}$ et al (2008) Female and male antisocial trajectories: from childhood origins to adult outcomes. Dev Psychopathol 20:673-716
8. Passamonti L, Fairchild G, Goodyer IM, Hurford G, Hagan CC, Rowe JB, Calder AJ (2010) Neural abnormalities in early-onset and adolescence-onset conduct disorder. Arch Gen Psychiatry 67:729-738

9. Fairchild G, Hagan CC, Walsh ND, Passamonti L, Calder AJ, Goodyer IM (2013) Brain structure abnormalities in adolescent girls with conduct disorder. J Child Psychol Psychiatry 54:86-95

10. Waschbusch DA, Carrey NJ, Willoughby MT, King S, Andrade BF (2007) Effects of methylphenidate and behavior modification on the social and academic behavior of children with disruptive behavior disorders: the moderating role of callous/unemotional traits. J Clin Child Adolesc Psychol 36:629-644

11. Blair RJR (2013) The neurobiology of psychopathic traits in youths. Nat Rev Neurosci 14:786-799

12. Brouns BHJ, de Wied MA, Keijsers L, Branje S, van Goozen SHM, Meeus WHJ (2013) Concurrent and prospective effects of psychopathic traits on affective and cognitive empathy in a community sample of late adolescents. J Child Psychol Psychiatry $54: 969-976$

13. Fairchild G, van Goozen SHM, Calder AJ, Goodyer IM (2013) Research review: evaluating and reformulating the developmental taxonomic theory of antisocial behaviour. J Child Psychol Psychiatry 54:924-940

14. Kretschmer T, Hickman M, Doerner R, Emond A, Lewis G, Macleod J, Maughan B, Munafo ‘ MR, Heron J. (2014) Outcomes of childhood conduct problem trajectories in early adulthood: findings from the ALSPAC study. Eur Child Adolesc Psychiatry (this issue)

15. National Institute for Health and Care Excellence (2013). Antisocial behaviour and conduct disorders in children and young people: recognition, intervention and management. NICE Clinical Guideline CG 158, National Institute for Health and Care Excellence, London. Available from: http://guidance.nice.org.uk/ CG158

16. Coghill D (2013) Do clinical services need to take conduct disorder more seriously? J Child Psychol Psychiatry 54:921-923

17. Zuddas A, Zanni R, Usala T (2011) Second generation antipsychotics (SGAs) for non-psychotic disorders in children and adolescents: a review of the randomized controlled studies. Eur Neuropsychopharmacol 21:600-620

18. Matthys M, Vanderschuren LJMJ, Schutter DJLG, Lochman J (2013) Impaired neurocognitive functions affect social learning processes in oppositional defiant disorder and conduct disorder: implications for interventions. Clin Child Fam Psychol Rev 15:234-246

19. Glennon J, Purper-Ouakil D, Bakker M, Zuddas A, Hoekstra P, Schulze U, Castro-Fornieles J, Santosh PJ, Arango C, Kölch M, Coghill D, Flamarique I, Penzol MJ, Wan M, Murray M, Wong IC, Danckaerts M, Bonnot O, Falissard B, Masi G, Fegert JM, Vicari S, Carucci S, Dittmann RW, Buitelaar JK; The PERS Consortium. (2013) Paediatric European Risperidone Studies (PERS): context, rationale, objectives, strategy, and challenges. Eur Child Adolesc Psychiatry. doi:10.1007/s00787-013-0498-3 